\title{
Revista Colombiana de Obstetricia y Ginecología
}

¿RIANO DE LA SOCIEDAD COLOMBIANA DE OBSTETRICIA Y GINECOLOGIA

Adeinistradu: JLAN N. BAQUERO - Apartado Nocional 270 - Correre 7a número 12-80-Oficina No. 23

Giumen II - Bogotá, Colombia - Agosto - Septiembre de 1951 - Número 5

\section{I P II R II B}

presentado por el inctor Rudulfo Camero, a la Sociedad de tiinecologia y Obstetricia, sobre las actividades cientificas desarrolladas en su jira por Sur América, Furopa y los Estados Unidos

Señor Presidente y demás miembros de la Directiva, señores colegas:

La Sociedad de Obstetricia y Ginecología me dió una carta, firmada por el doctor Rafael Ramírez, Presidente en esa época y con ella me presenté a las Sociedades similares en los países de habla castellana, visitados durante el viaje que acabo de realizar.

Pude observar que ai establecer o renovar estos vínculos se percibe un beneficio profesional y se deja un estímulo, que agregado al de otros colegas que viajen también, nos hará conocer un poco más.

En Lima, el Presidente de la Sociedad era, en marzo de 1950, el doctor César Heraud y lo fue también en esos mismos d'us, del Congreso Internacional de Cirugía en su cuarta reunión anual. Fuí invitado por su directiva para representar a Colombia y pude apreciar la maravillosa organización que le dieron $\mathrm{y}$ lo selecto de los trabajos presentados.

En Santiago de Chile la Sociedad me recibió con ocasión del cambio de Directiva. El doctor Manuel Avilés, Profesor agregado de Obstetricia, entregaba la Presidencia al doctor Héctor Cruz, agregado de Ginecología. Al día siguiente, y por invitación del doctor Cruz, fuí al Hospital San Vicente para visitar el servicio de Ginecología. Allí conocí al Profesor Juan Wood y encontré una magnífica organización que cuenta con laboratorio de Endecrino. logía, dirigido por el doctor Walter Cock. Contiguo a éste, estả el lahoratorio de química, luégo el de anatomía patológica y servi cio de Rayos $\mathbf{X}$. Tienen una biblioteca muy bien presentada y $e^{?}$ archivo de historias clínicas desde el año 1887. 
Por la noche asisti a la reunión de todo el cuerpo médico en el paraninfo de la Universidad Católsa, donde discutian los problemas con el Seguro Social. Sobre este punto trataré en el iriforme que rendiré a la Federación Médica.

Visité también el Hospital El Salvador, donde conoci al doc. tor Eduardo Bunster, Agregado también de Ginecología. Me manifestó complacencia por encontrar un médico colombiano, ya que precisamente estaba en consecución de unos datos sobre Colombia, únicos que le faltahan para una obra que tenia en pre. paración. Los datos eran los siguientes: Quién hizo en Colombia la primera persuflación simple. la primera quimografica, y lis primera histerosalpingogratia. Le respondi que como se trataba de datos estadísticos para un libro próximo a publicarse, me parecia más lógico y de más responsabilidad, que esos datos fueran suministrados oficiamente por la Sociedad de Ginecosogia y Obstetricia de Colombia. Encontró razonable mi argumento y le pro. meti escribir inmediatamente a Bogotá, como efectivamente lo hice, dando la dirección del doctor Bunster para que le enviaran estos datos. Un año después, recibí en Nueva York una carta dt el. en la que me decia su pesar por no haber recibido la menor información.

Encontré en los distintos hospitales y grupos de especialitas un gran entusiasmo por auxiliar la ginecología con estudio anatomopatológicos, endocrinológicos y radiológicos, en todos los casos. Asimismo, un gran interés por asistir a los congresos cien. tificos internacionales.

En la ciudad de Buenos Aires el interés por el estudio en to. das las ramas de la medicina, por las reuniones científicas en los hospitales y en la Asociación Médica Argentina, llega al máxino.

Alli tienen los médicos, en la Avenida Santa Fe, una de las principales, un edificio de dos pisos ocupado en su totalidad por dos salones dedicados exclusivamente a las reuniones semanat. de las distintas sociedades de especialistas. Cada salón tiene su equipo de proyecciones, amplificadores y negatoscopios, asi $40 m$ una silletería confortable y sistema de acústica muy bien acon. dicionado.

En todos los hospitales hay conferencias de las cinco de la tarde en adelante hasta las once o doce de la noche, sobre diver. sos temas y en distintos servicios. El Hospital Rivadavia cuentia, por ejemplo, con un servicio de Endocrinología, dirigido por el Dr. Enrique B. del Castillo, uno de los hombres más preparados en este ramo, quien posee una maravillosa dicción, grandes dote; de expositor y tiene entrenado un equipo de adjuntos capaces de dictar la conferencia cuando él está ausente o les cede la exposi- 
ción. Tuve oportunidad de admirarlo durante los cuatro meses que alli permanecí, pero más convencido quedé de su preparación. al encontrarlo citado en la bibliografía de las mejores revistas y libros de Europa y los Estados Unidos.

En el mismo Hospital Rivadavia funciona un servicio de esterilidad a cargo del doctor Guillermo Di Paola, agregado a la Cátedra de Ginecologia. Alli asisti durante mi permanencia en aquella ciudad. También hice estudios de Colposcopia con el docwor Alfredo Jakob. Director de la Maternidad Ramón Sardá Como usteder saben muy bien. este estudio fue iniciado por Hinselmann en Alemania y figura como gran auxiliar, junto con la técnica de Papanicolau en el diagnóstico precoz del cáncer del cuello uterino y para el diagnóstico diferencial de las distintas lesiones que alli se localizan.

En el Hospital de Clínicas y en el servicio de ginecologia a cargo del Profesor Juan C. Ahumada, uno de los cirujanos más anatómicos que conoci en toda mi jira, se hacen conferencias sobre Ginecología y Obstetricia para médicos graduados y se reúne también la Sociedad de Esterilidad.

En aquella ciudad encuentra el médico una de las mejores oportunidades para ampliar sus conocimientos, pues es realmente admirable el espiritu de trabajo y estudio que domina a la casi totalidad de los médicos. No hay congreso internacional al que no asistan. cuando menos, de cinco a diez delegados argentinos.

Cualquier época del año, a excepción de diciembre, enero y febrero, por el verano, es buena para asistir a los hospitales, y con un presupuesto de cinco dólares diarios se puede vivir en condiciones de relativa comodidad.

En Río de Janeiro visité al doctor Arnaldo de Morais en su servicio de Ginecología, donde también están haciendo estudios de Colposcopia. Allí supe del desarrollo que ha tomado la investigación centifica en Sao Paulo, circunstancia que es muy tenida en cuenta tanto en Europa como en los Estados Unidos.

De Europa haré un resumen lo más breve posible

En París asistí al Congreso Internacional del Cáncer, reunido en la Sorbona, y tuve la oportunidad de conocer destacados científicos de todo el mundo. Con gran satisfacción pude ver que una de las sesiones de aquel congreso era presidida por el doctor Alfonso Esguerra Gómez. Pasé luégo a Londres y de allí a Estocolmo, donde tenía el propósito de asistir al Radiumhemmett para conocer los tratamientos del cáncer uterino, que, como ustedes saben, figuran entre las técnicas más avanzadas del mundo. Allí conocí médicos que venian del Asia, del resto de Europa y de 
toda la América, con el mismo fin. Este Instituto del Cancer depende del Hospital Karolinska, que como todos los de aquella ciudad están dotados con toda la técnica y todo el confort. El director del Radiumhemmett es el doctor Hans Ludvig Kotmeier, hombre de profundos conocimientos, habla inglès. sueco, francés y alemán. Lo caracteriza una gran jovialidady se deleita enseñando $\mathrm{y}$ atendiendo a los médicos que lo visitan. El Profesor de Ginecologia en el Karolinska es el doctor Axel Westmann. tan competente como amable.

Allí en Suecia confirmé la idea inculcada desde Chile y la Argentina de que el urólogo, el patólogo y el radiólogo deben ser. los colaboradores inseparables del ginecologo y el obstetra, y que los especialistas en estas dos ramas debemos tener conocimientos bastante avanzados de aquellas otras. Para confirmar esta afirmación me referiré muy de paso al caso de una paciente colombiana a quien hemos tratado varios especialistas de esta Sociedad, para lesiones francamente ginecológicas y que hasta hoy se puede asegurar, que ha sido curada por el ginecólogo Westmann desde el terreno de la urología. Las molestias más frecuentes de dicha paciente eran hemorragias, cólicos pelvianos, flujos $y$ frecuentes elevaciones de temperatura, con otros sintomas de marcada importancia. Mediante radiografías y cistoscopias, el doctor Westman pudo localizar la saculación de un ure. tere en su desembocadura en la vejiga. Aquella retención ten:poral y periódica producía filtraciones al tejido vesical con todas sus repercusiones, $\mathbf{y}$ fuc ratada con termocauterio para los focos infecciosos y retracción de la mucosa, cateterismos del uretere. controlando todo esto por cistoscopias repetidas.

También se hacen actualmente estudios en el Hospital Sabasberg para el diagnóstico radiológico de la tuberculosis de las tompas.

A mi regrese a Paris visité las consultas externas de Gine. ologia del Hospital Broca, de L Hotel Dieu y asistí a un congre. n de esterilidad en el cual el doctor Raoul Palmer dictó sus con. ferencias sobre frigidez.

En el resto de Europa sólo me detuve para estudios ginecoĹgicos en Alemania con el Profesor Martius en la ciudad de Cottingen, en Viena can el Profesor Antoine, y en Madrid pude admirar a José Botella Llusía y Jesús García Orcoyen, los dos Irofesores de la Cátedra de Ginerologia. También asisti a unas conferencias sobre Endorinologia, de don Gregorio Marañói.

En Europa ya todos conocen la época en que se puede harer estudios pero en Suecia especialmente, sólo sería aprovechable sic abril a septiembre por causa de los fuertes inviemos. El cosio 
diario puede ser de seis dólares para los gastos de comida y alojamiento. El idioma inglés permite todas las informaciones en las ciudades principales.

Ya en lo Estados Unidos escogi la ciudad de Nueva York para el estudio de los temas que más me interesaban. Así fue como asisti al New York Hospital por espacio de seis meses, al servicio de Ginecología que dirige el doctor Gordon R. Douglas. Al Memorial Hospital para ver la cirugia de cáncer ginecológico practicada por el doctor Brunkswig. durante cuatro meses. alter nando la asistencia con el primero. En este mismo hospital pude admirar durante este tiempo al doctor Frank E. Adair. cirujano de cáncer del seno, de reconocida fama. También asisti a la consülta externa de esterilidad en el Medical Center, en cuya dirección está el doctor Buxton. En el Margaret Sanger Research, institución dirigida por el doctor Stone y dedicada exclusivamente al estudio de la fertilidad matrimonal, pude seguir los tratimientos que en líneas generales están siguiendo en todos los hospitales de los Estados Unidos.

En el New York Hospital encontré una organización del servicio de Ginecología y Obstetricia, establecida en forma tal, que todo el trabajo clinico $y$ hospitalario, junto con los laboratorios $y$ la consulta externa, permite la rotación del personal médico y da oportunidad de trabajo a una gran cantidad de especialistas. Esto, como salta a la vista, facilita la divulgación de los conocimientos más recientes $y$ despierta una gran atracción entre los médicos y por consiguiente gran estímulo para la investigación. Todos los lunes a las dos de la tarde se reúne el personal del servicio para presentar las historias clínicas de los casos más interesantes. Nunca se concibe reunión de esta clase sin la presencia del pato. logo. Allí tienen acceso y participación los médicos visitantes y en todas estas reuniones los médicos colombianos pasamos angustias cuando se nos piden las estadísticas que se llevan ayui sobre los casos que se discuten. Llama particularmente la aterción el que siendo la reunión a las dos de la tarde, cinco minutos antes ya no se encuentra sitio disponible, y si quien preside lit reunión llega dos minutos después de la hora fijada pide excusas a los asistentes. Durante seis meses, sólo en una ocasión pude registrar este retardo. Alli solicité al doctor Douglas una residencia para un médico colombiano y me manifestó buena volun. tad para cuando el candidato estuviera listo.

En el Memorial Hospital solicité dos residencias para médicos colombianos y el director clínico en ese entonces. doctor Whipple, me manifestó que no era dif́ícil mediante algunos re quisitos no difíciles de llenar. 
El entrenamiento quirúrgico que se puede obtener alli, es de una evidencia notoria, pues en el mismo piso hay ocho salas de cirugía en las cuales se está operando todos los días, desde las wcho de la mañana, y el visitante puede escoger la clase de cirugía que le interese. Para el residente es aún de mavor provecho. pues lo hacen zotar con todos los cirujanos cada dos meses. Los servicios de consulta externa, radiología y radiumterapia, son muy concurridos y aportan un material de practica de provecho incalculable.

En todos los hospitales se es bien recibido y todas las puertas se abren desde el momento en que se dan cuenta de que el interesado asiste diariamente. Allí se puede pasar el día entero para asistir a todos los trabajos, pudiendo hacer descansos en la biblioteca, donde llegan a diario las revistas más interesantes de todo el mundo, menos de Colombia.

Quiero informar a los colegas de la Sociedad. que traigo algunos equipos de actualidad que facilitan la labor de la especialidad y que próximamente tendré oportunidad de ponerlos a su disposición. Dejaré para otra reunión algunos proyectos que quiero someter a su consideración, insinuándoles esta noche tan sólo, la inclusión entre los socios honorarios o correspondientes que tenga ya la Sociedad, de los nombres de algunos especialistas que he conocido y que a juicio de ustedes puedan ser aceptados. Muchos de los socios actuales tienen también vínculos eon colegas de otros países y sería muy interesante hacerles la mis. ma comunicación. La ventaja de estas vinculaciones las aprovecharán los que en el futuro salgan a renovar teorías.

Señor Presidente y señores de la Directiva,

R. CAMERO C 